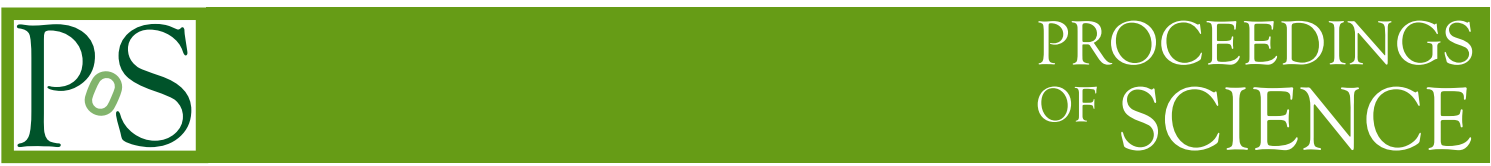

\title{
Supermodels: Early new physics at the LHC?
}

\section{Zoltan Ligeti*}

Lawrence Berkeley National Laboratory, University of California, Berkeley, CA 94720, USA

E-mail: ligetialbl.gov

\begin{abstract}
We investigate new physics that could be discovered with very little LHC data, beyond the expected sensitivity of the Tevatron. We construct "supermodels", for which the LHC sensitivity with $10 \mathrm{pb}^{-1}$ luminosity is already greater than that of the Tevatron with $10 \mathrm{fb}^{-1}$. The simplest supermodels involve $s$-channel resonances in the quark-antiquark and especially in the quarkquark channels. In the latter case, the LHC sensitivity with $0.1 \mathrm{pb}^{-1}$ can already be greater than that of the Tevatron with $10 \mathrm{fb}^{-1}$. We concentrate on easily visible final states with small standard model backgrounds, and find that there are simple searches, besides those for $Z^{\prime}$ states, which could discover new physics in early LHC data. Many of these are well-suited to test searches for "conventional" models, often discussed for larger data sets.
\end{abstract}

35th International Conference of High Energy Physics - ICHEP2010

July 22-28, 2010

Paris France

${ }^{*}$ Speaker. 


\section{Introduction}

We would like to explore what is the minimal luminosity the LHC needs in order to possibly discover new physics beyond the sensitivity of the Tevatron [1]. This talk concentrates on which new physics signatures could be discovered with a few $10 \mathrm{sof}^{-1}$ luminosity, beyond the reach of the $\sim 10 \mathrm{fb}^{-1}$ Tevatron data expected by the end of 2010 .

The definition of a supermodel in the Merriam-Webster Dictionary is "a famous and successful fashion model" - we define it as the class of models / Lagrangians which the LHC can discover with small luminosity, satisfying the following criteria:

1. Large enough LHC cross section to produce at least 10 signal events ${ }^{1}$ with $10 \mathrm{pb}^{-1}$ of data;

2. Small enough Tevatron cross section to evade the 2010 Tevatron sensitivity with $10 \mathrm{fb}^{-1}$;

3. Large enough branching fraction to an "easy" final state with essentially no backgrounds;

4. Consistency with other existing bounds.

However, we are not concerned with solving usual model building goals, such as unification, weak scale dark matter, or the hierarchy problem. Thus we got some criticism, e.g., that "Unfortunately, the defining property of supermodels is that they are unattainable" [2]. So it was amusing to see after this presentation the first ATLAS [3] and CMS [4] search results for resonances in the $q q$ channel, disproving this statement by obtaining limits that went beyond the Tevatron bounds for the first time. In addition, a special presentation by N. Sarkozy [5] was announced soon after this talk, who also disproved the claimed unattainability of supermodels using different techniques.

\section{Resonance scenarios}

To determine if a new physics scenario can be a supermodel, we need to compare $[\mathscr{L} \times \sigma \times$ $\mathscr{B} \times \mathscr{E}]$ at the LHC and the Tevatron, where $\mathscr{L}$ is the luminosity, $\sigma$ is the cross section, $\mathscr{B}$ is the branching ratio into a detected mode, and $\mathscr{E}$ is the efficiency. Since the Tevatron and LHC detectors are similar, to get a rough estimate, we can take $\mathscr{E}_{\mathrm{LHC}} \approx \mathscr{E}_{\mathrm{TEV}}$. If we further assume that the detection modes are the same, then $\mathscr{B}_{\mathrm{LHC}}=\mathscr{B}_{\mathrm{TEV}}$, and we simply need $\sigma_{\mathrm{LHC}} / \sigma_{\mathrm{TEV}}>$ $\mathscr{L}_{\mathrm{TEV}} / \mathscr{L}_{\mathrm{LHC}}$, i.e., $\mathscr{O}(1000)$ times larger LHC than Tevatron cross sections.

The cross section of any process is given by

$$
\frac{\mathrm{d} \sigma}{\mathrm{d} \hat{s}}=\sum_{i j} \hat{\sigma}_{i j}(\hat{s}) \mathscr{F}_{i j}(\hat{s}, s), \quad \mathscr{F}_{i j}(\hat{s}, s)=\int_{0}^{1} \mathrm{~d} x_{i} \mathrm{~d} x_{j} f_{i}\left(x_{i}\right) f_{j}\left(x_{j}\right) \delta\left(1-x_{i} x_{j} s / \hat{s}\right),
$$

where $i, j$ denote initial partons, $\hat{s}$ is the partonic center-of-mass energy while $s$ is that of the collider, $f_{i}\left(x_{i}\right)$ denote the parton distribution functions (PDFs), and $\mathscr{F}_{i j}(\hat{s}, s)$ is called the parton luminosity. If one partonic $i j$ channel and a narrow $\hat{s}$ range dominate, then $\sigma_{\mathrm{LHC}} / \sigma_{\mathrm{TEV}} \simeq$ $\mathscr{F}_{i j}\left(s_{\mathrm{LHC}}, \hat{s}\right) / \mathscr{F}_{i j}(2 \mathrm{TeV}, \hat{s})$. The dominance of a narow $\hat{s}$ range is quite generic, and is only a mild assumption for a first discovery, because the PDFs are steeply falling in the region relevant when only a few events are produced yet. We plot in Figure 1 the ratios of parton luminosities at the LHC and the Tevatron. We used the CTEQ-5L PDFs [6] implemented in Mathematica and checked that MSTW 2008 [7] gives compatible results at the level of accuracy we require. We see that for large enough $\hat{s}$, the parton luminosities at the LHC are indeed $>1000$ times larger than at the Tevatron.

\footnotetext{
${ }^{1}$ While fewer events may be sufficient for discovery, we demand 10 to allow for $\mathscr{O}(1)$ uncertainties in our analysis.
} 


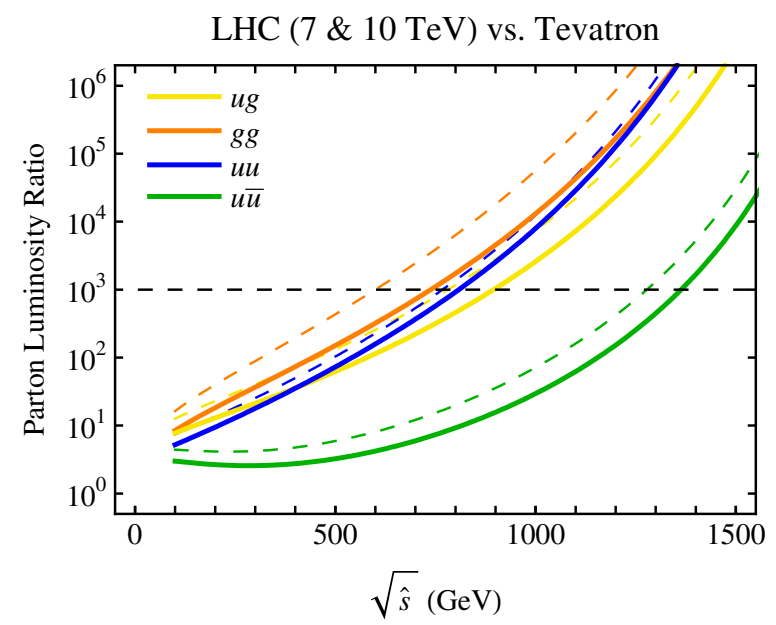

Figure 1: Ratios of the parton luminosities for $7 \mathrm{TeV}$ (solid) and $10 \mathrm{TeV}$ (dashed) LHC compared to the $1.96 \mathrm{TeV}$ Tevatron, as functions of the partonic invariant mass. The dashed horizontal line shows $10^{3}$.

To identify possible supermodel scenarios, we need to consider, besides the parton luminosities, the production rates and the decay rates to visible channels. In general, smaller production rates and smaller detectable branching ratios favor the Tevatron, because requiring enough events at the LHC for a discovery reduces the $\hat{s}$ values probed, thereby reducing the LHC's advantage.

Figure 1 shows that at moderate values of $\hat{s}$, the $g g$ parton luminosity is the most enhanced (hence the LHC is often called a gluon collider). Then the simplest process is the pair production of new colored particles, $g g \rightarrow X \bar{X}$, which can indeed have $\mathscr{O}(\mathrm{pb})$ cross sections at the LHC, promising $\mathscr{O}(10)$ events with $\mathscr{O}\left(10 \mathrm{pb}^{-1}\right)$ data. However, even assuming that $X$ and $\bar{X}$ decay to highly visible final states, the LHC's sensitivity only surpasses the Tevatron's with $\mathscr{O}\left(50 \mathrm{pb}^{-1}\right)$ luminosity [1]. The main reason is that the same final state can also be produced from $q \bar{q}$ initial states, where the LHC's advantage is less. Since QCD pair production is well-studied in many specific new physics scenarios, and the advantage of the early LHC over the Tevatron can only be marginal, we do not consider it to be a supermodel.

The production of an $s$-channel resonance has the potential to be a supermodel if it has a large coupling to the partonic initial state, since the production cross section for a single resonance is enhanced over pair production by a phase space factor, $16 \pi^{2}$. A resonance can have $\mathscr{O}(1)$ dimension-4 couplings to $q \bar{q}$ and $q q$ initial states. However, for the $q g$ or $g g$ initial states, $S U(3)$ gauge invariance forbids renormalizable couplings to a single resonance. The lowest dimension operator for the $g g$ initial state is a dimension-5 operator, $\left[g_{s}^{2} /\left(16 \pi^{2} \Lambda\right)\right] X G_{\mu v} G^{\mu \nu}$. The coefficient has been estimated assuming perturbative physics at $\Lambda \sim 1 \mathrm{TeV}$, with the $1 /\left(16 \pi^{2}\right)$ factor coming from a loop. (For the $q g$ initial state, one can produce an excited quark via a coupling of the form $\left[g_{s}^{2} /\left(16 \pi^{2} \Lambda\right)\right] \bar{q} \sigma_{\mu v} G^{\mu v} X$, which can also arise only from a loop diagram in a perturbative scenario.) If there is $\mathrm{TeV}$-scale strong dynamics involving $X$, then the coefficients can be enhanced up to their naive dimensional analysis value, $g_{s}^{2} /(4 \pi \Lambda)$. However, such strong dynamics near the $\mathrm{TeV}$ scale is constrained by precision measurements, and we adopt the perturbative estimate $g_{\text {eff }}^{2} \sim$ $\left[1 /\left(16 \pi^{2}\right)\right]^{2}$ for both $g g$ and $q g$ resonances. These considerations rule out supermodel resonances coupling dominantly to $q g$ or $g g$ initial states [1]. (Of course, if one allows $\mathscr{O}(1)$ couplings, without 

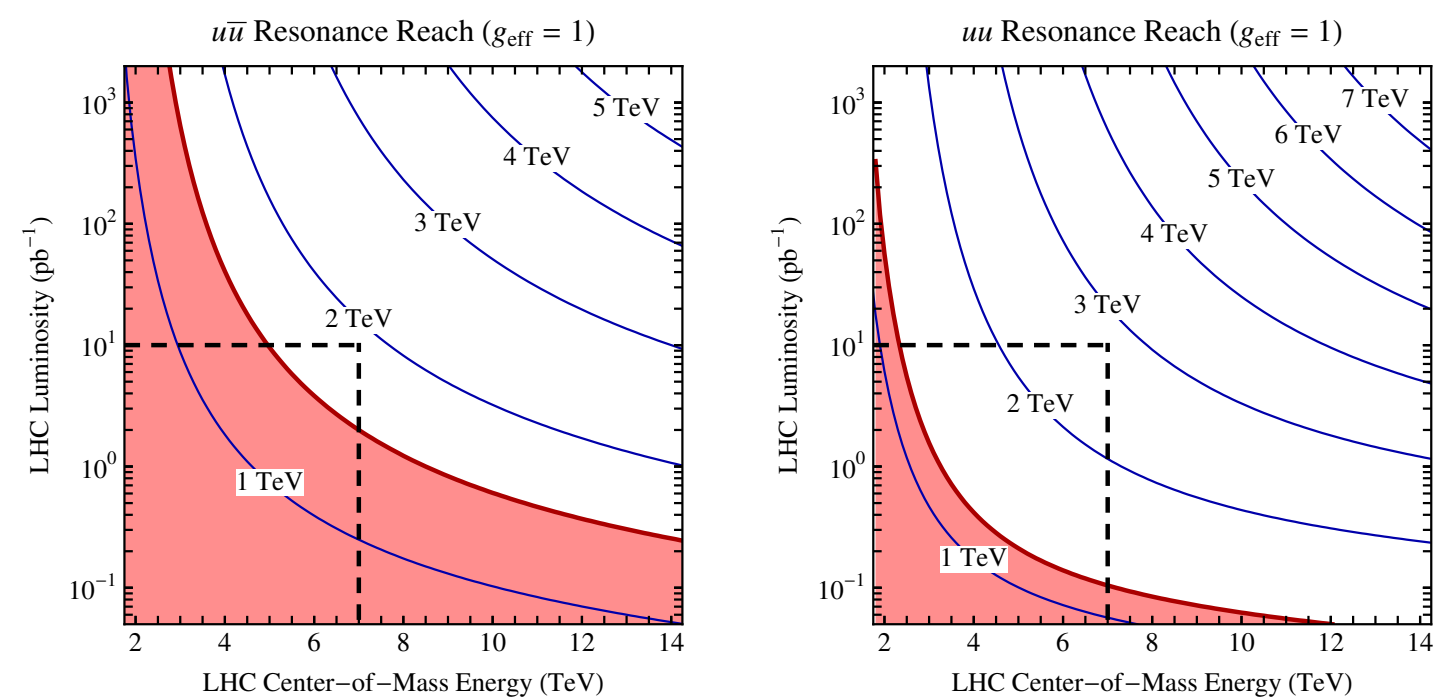

Figure 2: The LHC reach for resonance production, as a function of energy and luminosity. The contours show the production of 10 events for a given resonance mass. The red regions show the Tevatron sensitivity with $10 \mathrm{fb}^{-1}$, and the intersection of the dashed lines shows the maximal resonance mass probed by the $7 \mathrm{TeV}$ LHC with $10 \mathrm{pb}^{-1}$. The LHC exceeds the Tevatron sensitivity for $q q$ resonances already with $\sim 0.1 \mathrm{pb}^{-1}$.

the suppression factors suggested by naive dimensional analysis included here, then there are other possible supermodel resonance scenarios [8].)

Figure 2 shows our estimate of the generic early LHC reach in $m_{X}$, as a function of the energy and luminosity, for $q \bar{q}$ and $q q$ resonances, assuming $100 \%$ branching fraction to highly visible final states (for concreteness, for $q=u$ ), showing that such resonances can be supermodels. For simplicity, we call any $q \bar{q}$ resonance a $Z^{\prime}$ (even if it is a KK-gluon), and any $q q$ resonance a "diquark" (even if it is a colored scalar). Figure 2 shows that the LHC sensitivity for $q \bar{q}$ resonances surpasses the Tevatron with $\mathscr{O}(10) \mathrm{pb}^{-1}$ data. However, for $q q$ resonances, the LHC surpasses the Tevatron already with $\mathscr{O}(0.1) \mathrm{pb}^{-1}$ at $7 \mathrm{TeV}[1]$, as demonstrated experimentally at this Conference [3, 4].

\section{Supermodel building}

$q \bar{q}$ resonances: Until recently, the most often discussed new physics scenarios for early LHC discoveries have been $Z^{\prime}$ models. However, a model with a $Z^{\prime}$ coupling to leptons and quarks is strongly constrained by the LEP II limits on four-fermion operators. Flavor universal models face the problem that the production rate is proportional to the $Z^{\prime}$ coupling to quarks, $\sigma\left(q \bar{q} \rightarrow Z^{\prime}\right) \propto g_{q}^{2}$, while the branching ratio to $\ell^{+} \ell^{-}$is suppressed by it, $\mathscr{B}\left(Z^{\prime} \rightarrow \ell^{+} \ell^{-}\right) \propto g_{\ell}^{2} /\left(2 g_{\ell}^{2}+6 g_{q}^{2}\right)$. In this class of models there is no value of $m_{Z^{\prime}}$ for which the LHC can see $10 Z^{\prime} \rightarrow \ell^{+} \ell^{-}$events with $10 \mathrm{pb}^{-1}$, without violating other bounds [1]. This conclusion can be evaded by coupling the $Z^{\prime}$ only to muons, studied in detail also in Ref. [9]. A $B-3 L_{\mu}$ boson is a supermodel, but it ain't pretty.

If other particles are introduced and the $Z^{\prime}$ decays with large branching fraction to non-SM particles then many possibilities open up. For example, a $q \bar{q}$ resonance decaying to new quasi-stable charged particles can have large branching ratios, avoid flavor physics bounds, and be cosmologi- 
cally safe. Another option is if the resonance decays to hidden-valey type states, which then have small couplings to decay back to SM particles; though this is unlikely to be easily reconstructible.

$q q$ resonances: As can be seen from Figure 2, enormous cross sections are possible for a resonance in the $q q$ channel, and the LHC sensitivity extends to several TeV. The simplest decay of such a resonance is back to two jets, and for a diquark at $2 \mathrm{TeV}$ (or above), its contribution to the two-jet rate is comparable with the QCD background. The flavor physics constraints, which could be severe, can be satisfied by making the diquark models minimally flavor violating [10]. As for a $Z^{\prime}$, if we introduce additional new particles, more spectacular signals are possible. Models can be constructed in which the final state is two new charged particles, or $2 j+\ell^{+} \ell^{-}$[1]. The latter final state is well-studied for $W_{R}^{ \pm}$searches, however, that has discovery potential only with $>1 \mathrm{fb}^{-1}$ data, whereas the same $2 j+\ell^{+} \ell^{-}$search for a diquark is already interesting with $>10 \mathrm{pb}^{-1}$.

\section{Conclusions}

We explored new physics "supermodel" scenarios that the LHC can discover with $\mathscr{O}\left(10 \mathrm{pb}^{-1}\right)$ data. With $1-10 \mathrm{pb}^{-1}, s$-channel resonances coupled to $q q$ initial states ("diquarks") are the most promising, while with more data $q \bar{q}$ resonance $\left(Z^{\prime}\right)$ searches also become interesting, especially decaying into new charged particles. While some supermodels may not be as attractive as the name suggests, the same final states are useful search channels for more conventional models later on.

Acknowledgments I thank C. Bauer, M. Schmaltz, J. Thaler, and D. Walker for a most enjoyable collaboration. This work was supported in part by the Director, Office of Science, Office of High Energy Physics of the U.S. Department of Energy under contract DE-AC02-05CH11231.

\section{References}

[1] C. W. Bauer, Z. Ligeti, M. Schmaltz, J. Thaler and D. G. E. Walker, Phys. Lett. B 690 (2010) 280 [arXiv:0909.5213 [hep-ph]].

[2] M. Peskin, talk at "The Search for New Particles and Forces" workshop at the GGI, Firenze, Italy, October 2009, http: / /www.ggi.fi.infn.it/talks/talk1376.pdf.

[3] G. Choudalakis, ATLAS Collaboration, talk at this Conference, http://indico.cern.ch/ contributionDisplay ·py?contribId=541\&conf Id=73513.

[4] K. Kousouris, CMS Collaboration, talk at this Conference, http://indico.cern.ch/ contributionDisplay py? contribId=847\& conf Id=73513.

[5] N. Sarkozy, talk at this Conference, http: / / webcast.in2p3.fr/2010/ichep/index.php?video=Sarkozy.ram.

[6] H. L. Lai et al. [CTEQ Collaboration], Eur. Phys. J. C 12, 375 (2000) [hep-ph/9903282]; and http: //www.phys.psu.edu/ cteq/.

[7] A. D. Martin, W. J. Stirling, R. S. Thorne and G. Watt, Eur. Phys. J. C 63, 189 (2009) [arXiv:0901.0002]; and http: / / projects.hepforge.org/mstwpdf/.

[8] R. Barbieri and R. Torre, arXiv:1008.5302.

[9] E. Salvioni, G. Villadoro and F. Zwirner, JHEP 0911, 068 (2009) [arXiv:0909.1320]; E. Salvioni, A. Strumia, G. Villadoro and F. Zwirner, JHEP 1003, 010 (2010) [arXiv:0911.1450].

[10] J. M. Arnold, M. Pospelov, M. Trott and M. B. Wise, JHEP 1001, 073 (2010) [arXiv:0911.2225]. 